\title{
Smart Electrical Design of Medical Center to Vary Field Parameters: Sensor Network in Improving Health Care
}

\author{
${ }^{1,2}$ Wadee Alhalabi, ${ }^{3}$ Aqeel Farooq, ${ }^{1}$ Ahad Alhudali and ${ }^{1}$ Lujain Khafaji \\ ${ }^{1}$ Department of Computer Science, Effat University, Jeddah, Kingdom of Saudi Arabia \\ ${ }^{2}$ Department of Computer Science, King Abdulaziz University, Jeddah, Saudi Arabia \\ ${ }^{3}$ Department of IT, Bright Inc., Jeddah, Kingdom of Saudi Arabia
}

\begin{abstract}
Medical center plays a vital and key role in our daily life. Without having medical centers, maintaining a healthy life is impossible. The key focus of this research is to strategize the smart electrical design for the medical center (using sensors and manageable user interfaces). Electrical design involves planning, creating, testing, managing, supervising, installing and operating the electrical devices and equipment such as lightning equipment, power systems, power distribution, fire and life safety equipment and data communications network installation. Inside the medical center, the field interference from the electrical appliances and electrical wiring effect the patient's healing capabilities. Using sensors that detect interference fields (electrical+magnetic) and circuitry to control the voltage level/current flow, an environment can be produced that is smart and in which patient can heal quicker. A network of sensors allows the user to actively interact with the environment. In this research, to reduce the effect of field on the patient healing abilities, medical center electrical design with DC voltage is proposed. This research work focuses on designing the smart sensors network to monitor the field produced by the electrical design in the medical center. Using the field data from the sensors, the DC voltage to the devices can be turned $\mathrm{ON} / \mathrm{OFF}$ or varied for variable resistive based devices. The proposed sensor scheme creates a wireless sensor environment as the sensors modules interact with each other wirelessly. This creates a smart environment fitted for medical research and patient instant healing.
\end{abstract}

Key words: Medical centers, lightning equipment, power system, power distribution, fire and safety equipment, healing capabilities, wireless sensor network

\section{INTRODUCTION}

We are living in a world that seems to be have more energy crisis than before. The traditional power grids, generation and distribution utilize and consume way more resources (Pimentel et al., 1973). This in turn, create great pressure on the already available resources and deteriorate them. This is not the only reason which urges us that to design the system efficiently as much as we can to save the burden on the current energy resources. Besides transmission line losses, wide-scale power blackout leaves millions of peoples, resources and services without electricity every year. These consideration helps in the understanding the importance of the careful design. Healthcare or medical center plays a key role in our life. In general, medical center are places where health care is provided. Places such as small clinics, doctor's offices, urgent care center, large hospitals, emergency rooms, trauma centers are considered as the part of medical center (Felton, 1998). Most of the countries around the world regulates license to the medical care centers. The license is given after complete survey by a governmental time. The governmental team checks for complete requirements such as electrical installation, electrical safety, electrical wiring, construction design of building, medical equipment, design of lightning, etc. Most of the governments have developed standards for the guiding the companies which helps in setting up the environment. Famous example of this standard chart includes, Australian and New Zealand standard guide for electricity, etc. The main aim of this thesis is to design the electrical systems for dental clinic.

Electrical design involves planning, creating, testing, managing, supervising and installing the electrical equipment (Smith et al., 1995). The installed equipment includes lightening equipment, power systems, fire and safety alarms (Smoke alarm) and other miscellaneous electrical equipment. Some clinics require communication equipment's as part of their apparatus. We are including dental clinic which involves X-ray machines with proper care as well. The simple example is connecting the flashlight cell through two wires to a light bulb and make it glow. The aim of engineers should be to design the system that operate on minimum energy resources (Casazza, 1978). Considering the most sophisticated definition, electrical design is preferably the design of

Corresponding Author: Wadee Alhalabi, Department of Computer Science, Effat University, Jeddah, Kingdom of Saudi Arabia 
(Casazza, 1978). Considering the most sophisticated definition, electrical design is preferably the design of various electrical systems. These includes proper wiring for providing electricity to electrical systems as well. Electrical systems are electrical components connected with each other to carry out specifically programmed operations. For example, a railway system contains power design for rail cars, railway station and connected loads. The process involves design the system at first and making it functional later. Electrical design is the most important consideration while creating the smart city and the smart economy. This is an era of digital communication and digital technology but without smart electrical design, it is not possible to achieve any means of smart technology.

The aim of this research is to design the electrical configuration such as electrical wiring, system diagram, machines placement and sensor arrangement for the medical center in such a way so that medical center act as smart center in terms of the proving medical treatments. The project thesis aims in providing the diagram for the medical center.

Literature review: Medical centers are the places where medical treatment is provided to patients. Medical centers are designed with utmost care to make sure that healthy environment will become available to patients. Electrical wiring is done in such a way that efficient control of it will become available to non-technical people. Also, the same care needs to be done while designing the electrical devices used in the medical center. It's been the part of different standards and their rules states that human must interact with a device after having a proper care applied and associated with it.

Regarding safety both the patients and the surgeon must follow the country's medical standards for their appropriate safety. Using the latest research, the internationally adopted standards are also improving day by day. For the electrical sensors, the word "transducer" is used universally. A sensor is a device that measures the physical quantity. There are different types of sensors available.

The main category is to select from analogue or digital. The type of sensors used depend on the physical quantity to be measured. There are numerous sensors commercially available in the market. Electrical sensors are the sensing devices that measure the physical quantity from the real world and the measurement of this quantity helps in the producing improvement in the measured physical parameter using actuating actions or at least help in showing the alert (Elsayed et al., 2017). Air conditioning systems are quite vital for engineers because it is counted as one of the biggest needs of construction of building, so, it is essential to see its aspects and identify its types. The question is how much power it consumed and acts as system load. Built on Heat Transfer that is identified heat is a confined form of energy. Each object in the globe has some heat energy associated with it. If the objects heat energy is less, then it can be said to has achieved the cold state. Conduction is the process of transferring heat from one object to another and it makes the body that transferred the heat cooler (Omer and Muhammad, 2017). When an air conditioning system refrigerates, it is actually eliminating heat and transfer it to the environment. This can be demonstrated by turning on a spot. Cooler and placing one hand in front of the cold air nozzle and the other over the warm air exhaust. The action of transfer of heat can be realized in this way.

Electrical devices are used to connect air conditioners and other miscellaneous operational devices to a smart place. Sockets and plugs often combine male and female aspects but the bare pins or terminals in the socket are not energized always used for fixed the plugs of load. AC power plugs and sockets are devices used to supply power to from the main source. A plug connects a device to a socket. Plugs are mostly or completely male while sockets are mostly or completely female the plug has pins that fit into matching slots or holes in the socket (Yingying et al., 2015). Wall-mounted sockets are also called outlets. In order to reduce the risk of electric shock, plug and socket systems are to join a variety of safety features. Sockets can be intended to accept only compatible plugs and reject all others unnecessary fitted one's. Some systems are designed with a technique to remove the dangerous unsafe voltage level or moreover the sensors are installed to detect such condition (Wan et al., 2010).

\section{MATERIALS AND METHODS}

Center design: Figure 1 shows the design diagram of the medical center. The medical center under consideration is designed with 4-rooms and one lobby. This clinic design is set for dental clinic. There are four rooms for clinical procedure and one lobby where registration desk can be done for patients. The design symbol for the constructional design are also a part of the design diagram and has been shown in Fig. 2-4. The medical center is designed to be compact and involved with air conditioner. The air conditioner is set to be a location from where impact cooling can be achieved. 


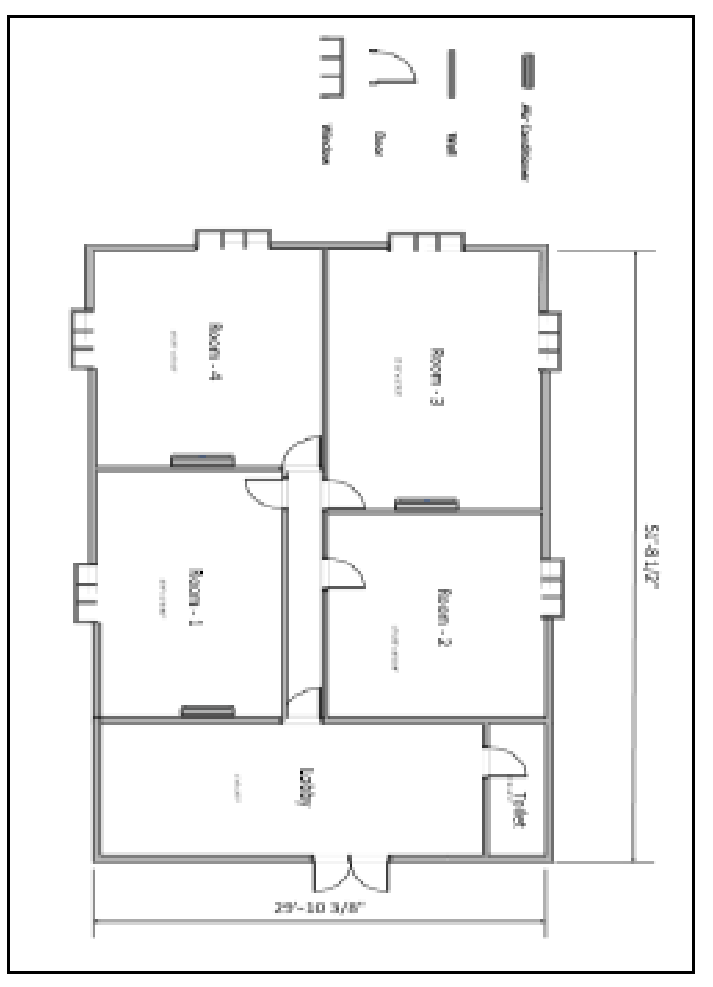

Fig. 1: Design of medical center (Visio diagram)

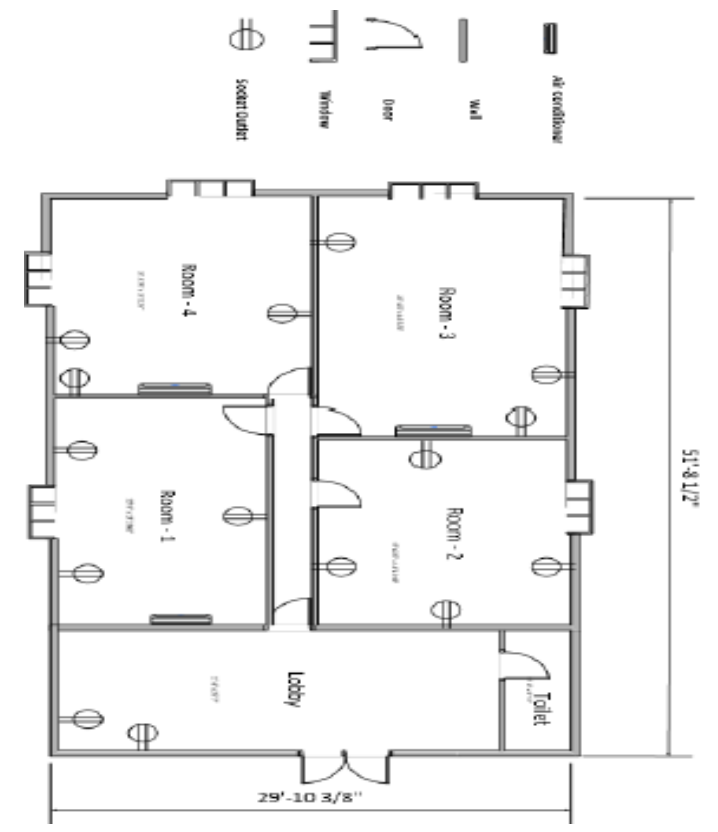

Fig. 2: Medical center with lightning

Air conditioner and lightening design: There are various types of Air Conditioning (AC) systems that are precisely intended for different types of applications. Some of the

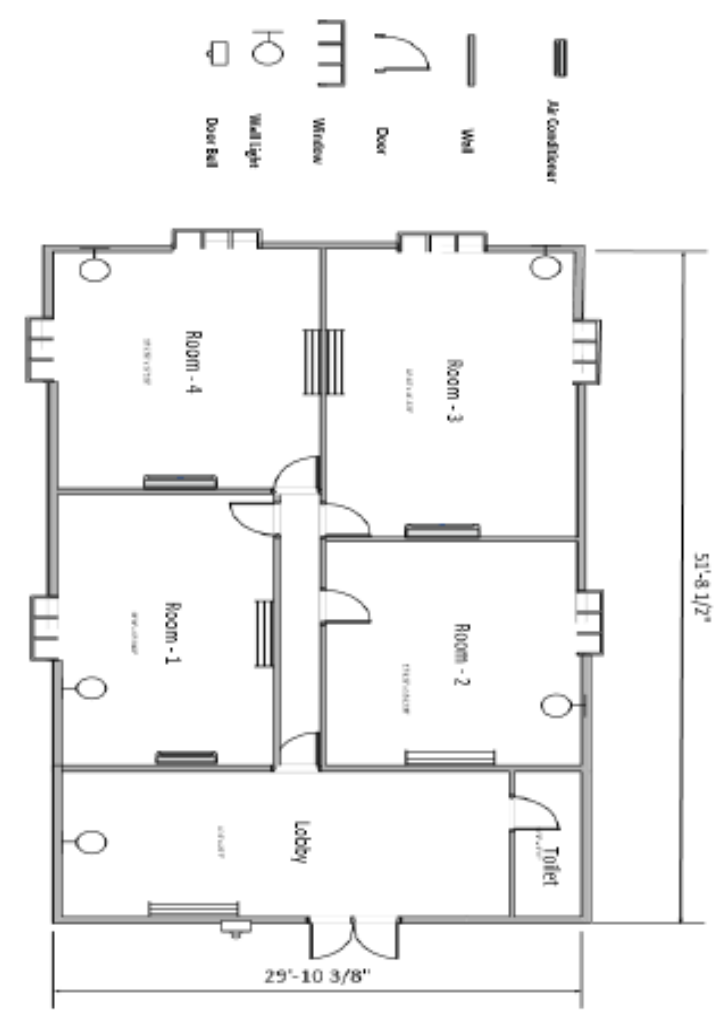

Fig. 3: Design with electric sockets

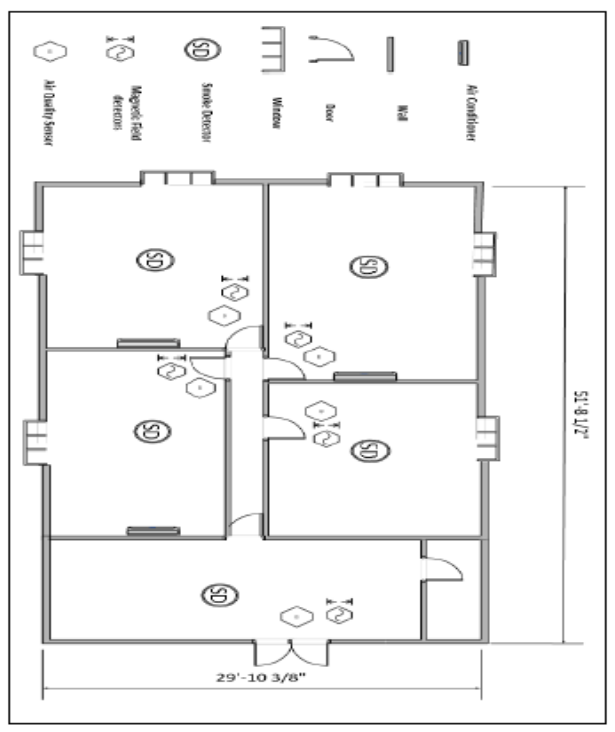

Fig. 4: Design with sensors placed in the center

below listed applications describe the uses of the different types of air conditioners. First step in the process is indicating an $\mathrm{AC}$ unit for location is to select the type of 


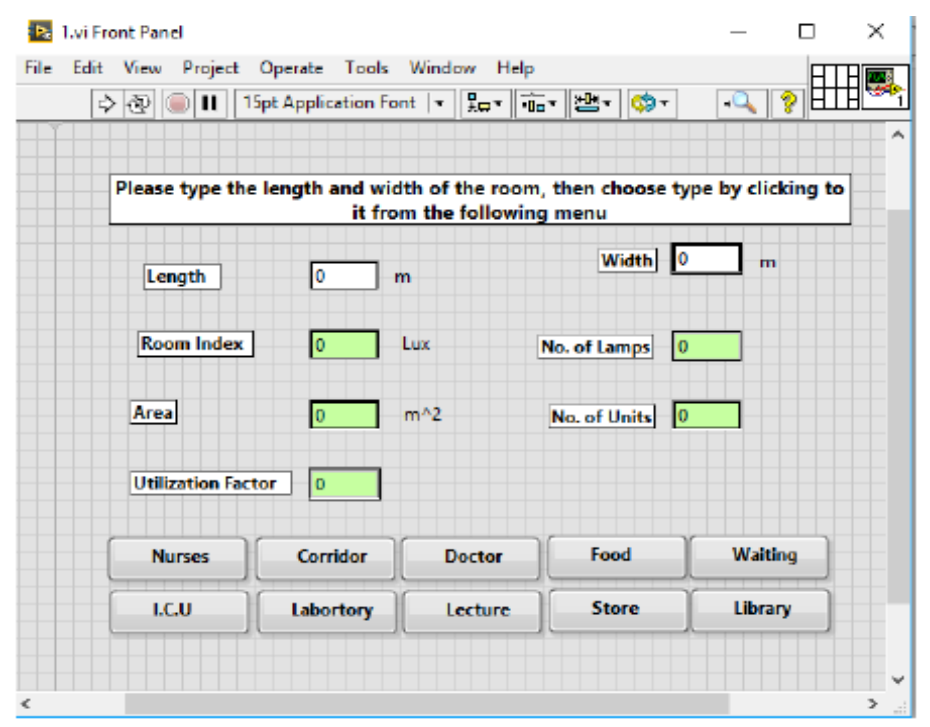

Fig. 5: Lightning system program

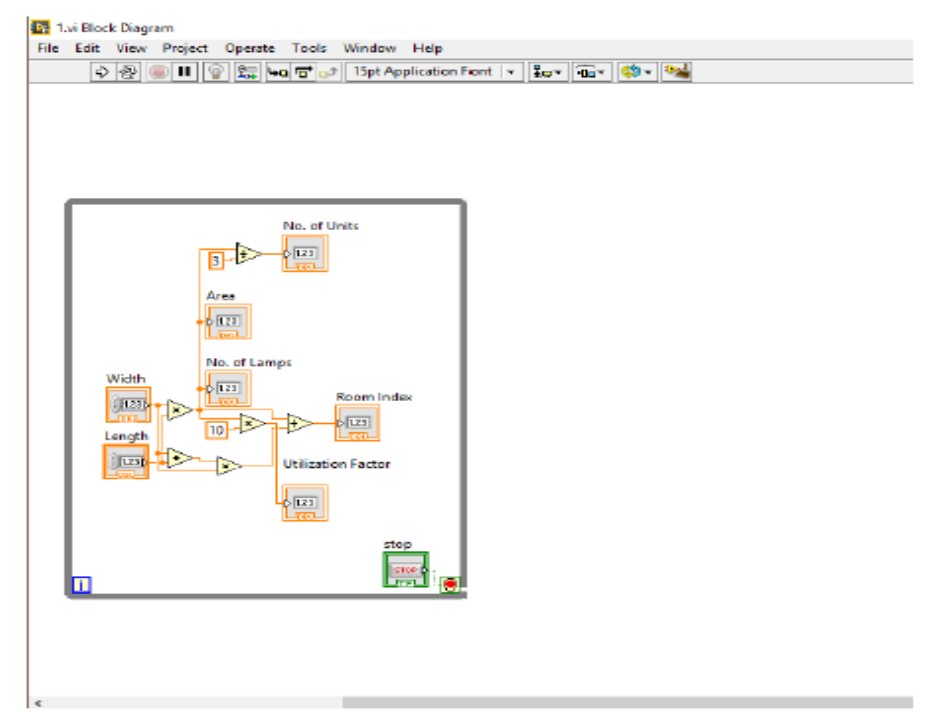

Fig. 6: LabVIEW block diagram for lightning program

the air conditioning unit. There are numerous types of $\mathrm{AC}$ units available commercially in the market. Window $\mathrm{AC}$ is the usually used $\mathrm{AC}$ for separate rooms as they utilize the air pressure as well. In this type of $\mathrm{AC}$, complete components such as condenser, compressors, coil, expansion valve and cooling rods are enclosed in a closed box. The AC unit is tailored in slot ended room wall (Haitao et al., 2006). Window AC are designed to fits in a place for home user. They are one of the most widely used types of AC because of their simplest form. Window AC encompasses of the solid base on which all the parts of it are accumulated. The base is accumulated privileged the casing which is perfectly fitted into the wall or the window of the apartment. The whole assemblage of the window $\mathrm{AC}$ can be divided into two components, room side (contains cooling side and outside side) (Koizumi et al., 1991). The split AC has two different parts: indoor and outdoor unit. The outdoor unit, fitted outside the room, houses parts such as condenser and compressor shown in Fig. 5 and 6.

Socket design: AC plug or electrical sockets are the devices that allow electrical devices to be connected with them and operate on $\mathrm{AC}$ power. A general electrical socket provides voltage to the connected device when device gets connected with electrical socket, it draws 


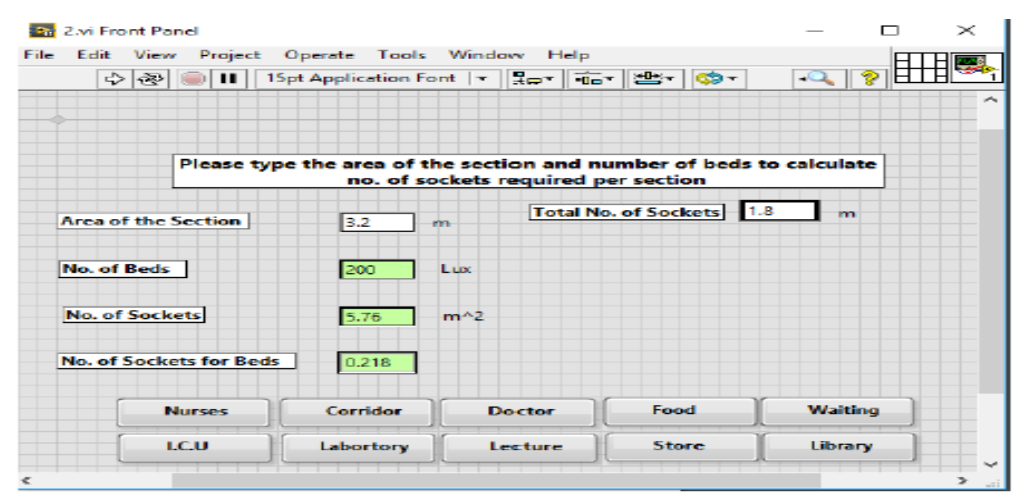

Fig. 7: Socket calculator

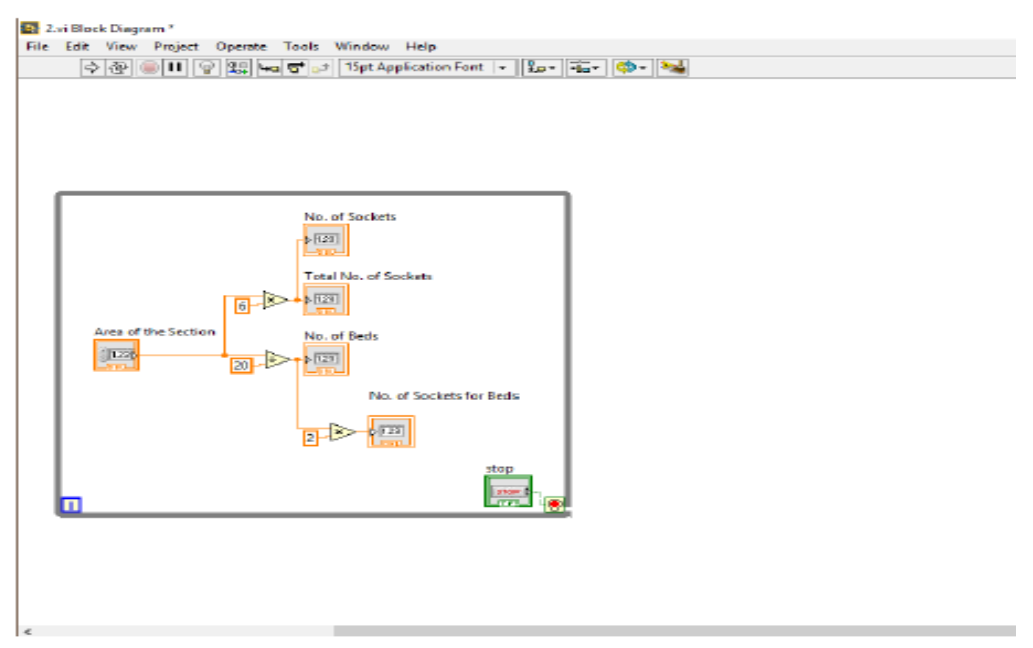

Fig. 8: LabVIEW block diagram for socket calculator

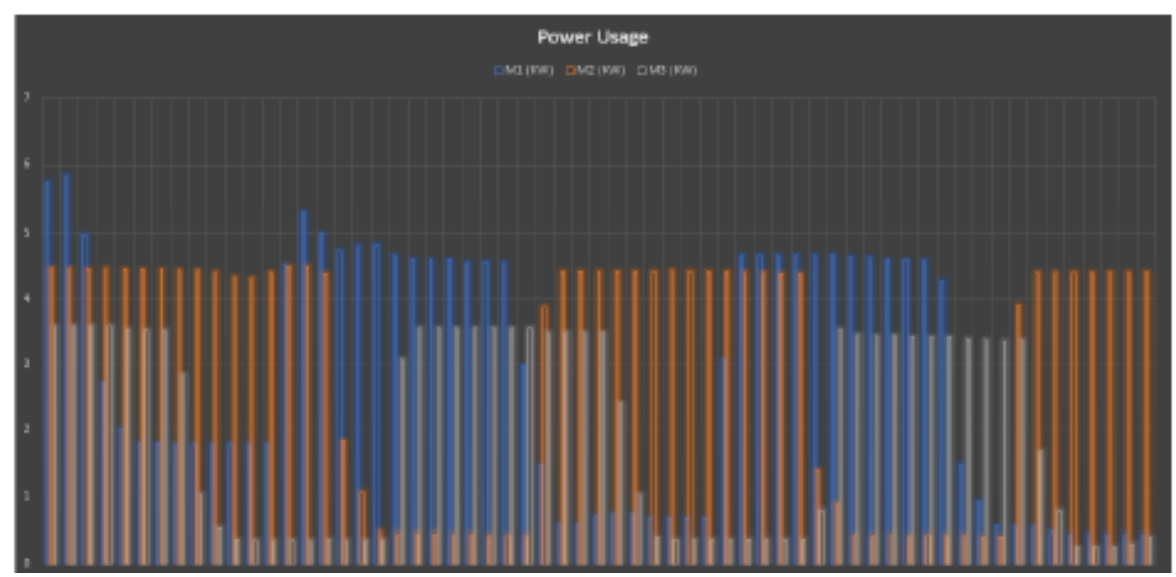

Fig. 9: Power usage for each of these models

voltage from the main supply. Electrical sockets are the general interface they are supply used to connect with devices. Each country has its own standard for designing and manufacturing the electrical sockets, some of them are listed in the IEC technical report TR 6600083 Fig. 7-9. The IEC TR 60083 states that the report only 


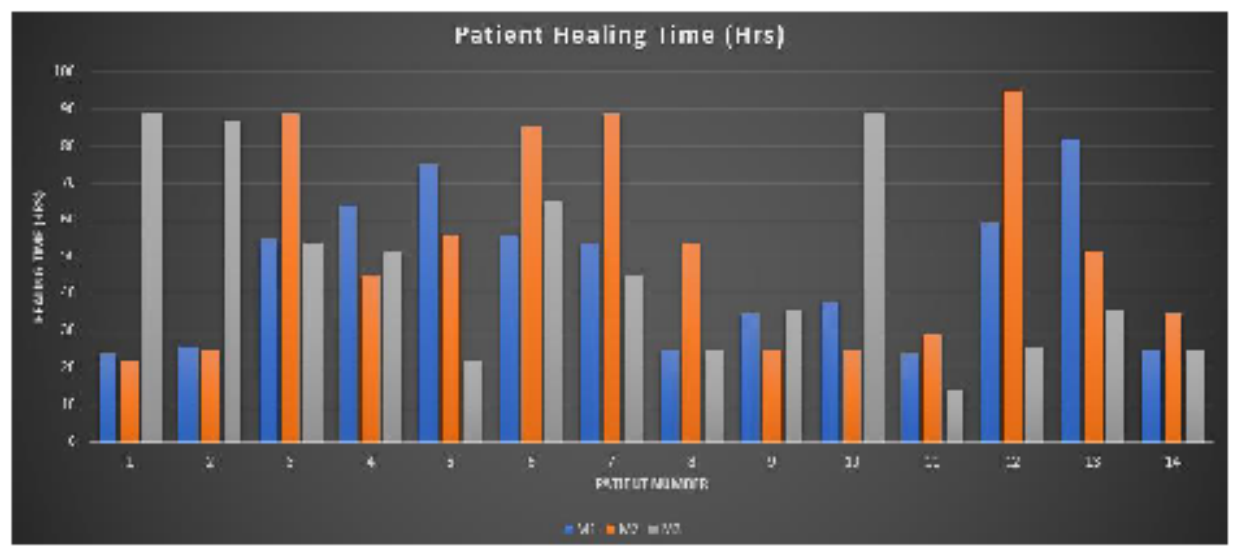

Fig. 10: Patient healing time with magnetic field

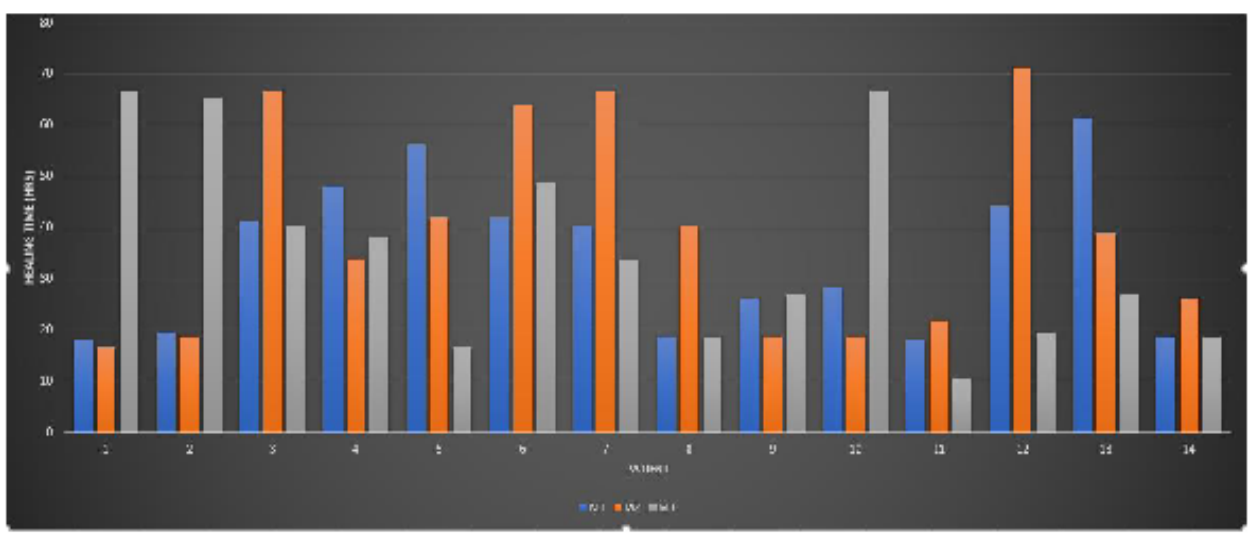

Fig. 11: Predicted healing time with reduced magnetic field

contains systems for which standard sheets have been published in National Standard which my a be a National Standard of the country itself or any other IEC member country. IEC standards are mostly adopted for designing electrical devices (Tsai et al., 2010) (Fig. 10 and 11).

Sensor design: Sensors are electrically transducer devices that measures the physical parameters and generates the equivalent voltage. In this experimental medical design thoughts are to use magnetic field sensors, Gas sensor and Air quality sensors to check for the equivalent valid availability of the certain quantity. Once the magnetic field goes beyond a certain range, the alarm will be generated. The smoke sensors will do the same. The air quality sensors equivalently check for the oxygen content in the room. For air quality detection, oxygen sensors have been used. Magnetic field presence stops the process of quick healing for the patient, so therefore, detection of magnetic field after the regular interval is required to generate alarm which helps useful for alerting patient and medical staff. Furthermore, the technology can be used to insert air pressure using oxygen cylinders that balances the medical center air pressure. The design of medical center with sensors positions and labeling.

\section{RESULTS AND DISCUSSION}

The simulations are performed in LabVIEW to develop a program that calculates the number of sockets and the lightning system (Badawood, 2016).

Lightning program: The graphical interface for calculating the lightning and sockets requirement has been developed in LabVIEW to calculate the currents required, cables and circuit breakers rating of each floor in the hospital for lighting system. Figure 7 shows the simulation snapshot of this program.

Socket program: using the LabVIEW, a program is developed to calculate the currents required, cables and 
circuit breakers rating of each floor in the hospital for AC socket. The screenshot of the running program. To do the testing for efficient design in terms of sun radiations that affects the system, three scenarios are used, these scenarios have been named as M1-M3. The data is collected from the three different medical centers in Saudi Arabia. The power usage for these cases has been shown in Fig. 10. The power data has been shown in $\mathrm{kW}$ for a 60 days from September to Oct for different medical centers from Saudi Arabia. These used clinics are the small medical clinics. One line shows the average power data for a day has been shown in Fig. 11.

More is the power, more will be the magnetic field because magnetic field is produced from the actuator used in the machinery. These machineries exist in almost all the devices available in the medical center. Large value of magnetic field around the patient reduces the healing capability. Less operated patient healing time in hours is shown for 15 patients. Based on the Henry results (Henry et al., 2008), healing time is predicted to decreased up to $25 \%$ of total time for all three scenarios. Figure 11 shows the healing time for random three scenario patients whereas the predicted healing time for the same patients based on the condition that preventive measure will be taken to reduce the magnitude of the magnetic field.

\section{CONCLUSION}

The work presented in this contains the main aspects that depicts and revolves the concept and usage of the latest electrical techniques for designing medical center. The design process points out several times what are the reason behind choosing the selected techniques while ignoring others. This at the same time highlights the design issue that occurs during the process. In the literature review section, some of the power providing sources have been discussed. This thesis work involves targeting the design diagram for the medical center (Dental clinic). The attention was then moved on the control strategies of a community, in particular on decentralized schemes. A simplified statistical design diagram framework has been presented in order to better contextualize the problem. The main problem that occurs during the design process is to investigate the correct positions for sensors, sockets, air conditioner and respective devices. There can be several possible combinations for placing the devices but in this thesis work only the best scheme has been selected which fulfills the requirement of the medical center in Saudi Arabia. Magnetic field sensor, oxygen sensor, smoke detectors are used to measure the environmental characteristics of the medical center. The problem study case or consideration point is that during the patient healing time period, the magnetic field inside the medical center effects then patient healing capabilities. The detection of the magnetic field inside the medical center helps in maintaining the safe magnetic field level in the medical center. Moreover, the constructional design, design with sensors placemen, design with sockets and lights have been shown as a part of thesis. A LabVIEW program has been developed that calculates the lightning system and sockets strength. Finally, knowing about the simultaneous actions of load in the medical centers, gives the central entity a better insight of the situation, making possible to put in place cost-effective strategies for the optimal power usage. Hierarchical control mechanism requires however expensive machinery and sharing personal information such as consumption habits and it is not that easy to find the optimal strategy with so many unpredictable parameters. The thesis involves the medical center design in which sockets, sensors, air conditioners placements is being discussed. The different power providing mechanism are also the part of this thes is researcher.

\section{REFERENCES}

Badawood, A.A., 2016. Electrical design for medical center. Intl. Adv. Res. J. Sci. Eng. Technol., 3: 69-72.

Casazza, J.A., 1978. The engineer's role in the energy crisis. Technol. Soc., 6: 5-9.

Elsayed, M., M. Mahmuddin, A. Badawy, T. Elfouly and A. Mohamed et al., 2017. Walsh transform with moving average filtering for data compression in wireless sensor networks. Proceedings of the 2017 IEEE 13th International Colloquium on Signal Processing and its Applications (CSPA), March 10-12, 2017, IEEE, Batu Ferringhi, Malaysia, ISBN:978-1-5090-1184-1, pp: 270-274.

Felton, J.S., 1998. Burnout as a clinical entity-its importance in health care workers. Occup. Med., 48: 237-250.

Haitao, A.Q., B.W. Yan and C.S. Shouqian, 2006. Green air-conditioner design. Proceedings of the 7 th International Conference on Computer-Aided Industrial Design and Conceptual Design CAIDCD'06, November 17-19, 2006, IEEE, Hangzhou, China, pp: 1-4.

Henry, S.L., M.J. Concannon and G.J. Yee, 2008. The effect of magnetic fields on wound healing experimental study and review of the literature. Eplasty, 8: 393-399. 
Koizumi, Y., K. Yotsumoto, H. Hayama and S. Furubo, 1991. Design considerations of power systems for the air conditioner used in telecommunication networks. Proceedings of the 13th International Conference on Telecommunications Energy INTELEC'91, November 5-8, 1991, IEEE, Kyoto, Japan, pp: 410-415.

Omer, S.A.U.R. and E. Muhammad, 2017. Design of intelligent air conditioner controller using fuzzy logic. Proceedings of the 2017 International Conference on Innovations in Electrical Engineering and Computational Technologies (ICIEECT), April 5-7, 2017, IEEE, Karachi, Pakistan, ISBN:978-1-5090-3310-2, pp: 1-5.

Pimentel, D., L.E. Hurd, A.C. Bellotti, M.J. Forster and I.N. Oka et al., 1973. Food production and the energy crisis. Sci., 182: 443-449.

Smith, R.W., I.L. Freeston and B.H. Brown, 1995. A real-time electrical impedance tomography system for clinical use-design and preliminary results. IEEE. Trans. Biomed. Eng., 42: 133-140.
Tsai, C.H., Y.W. Bai, C.A. Chu, C.Y. Chung and M.B. Lin, 2010. Design and implementation of a socket with zero standby power using a photovoltaic array. IEEE. Trans. Consum. Electron., 56: 2686-2693.

Wan, Z., H. Chen, K. Lin, T. Jin and Z. Zheng, 2010. Optimization design investigation of Volute tongue in indoor air-conditioner. Proceedings of the International Conference on Asia-Pacific Power and Energy Engineering (APPEEC), March 28-31, 2010, IEEE, Chengdu, China, ISBN978-1-4244-4812-8, pp: $1-4$.

Yingying, C., X. Xiuying, W. Ming and J. Haiming, 2015. Design of a control system for a fresh air conditioner. Proceedings of the 2015 27th International Conference on Chinese Control and Decision (CCDC), May 23-25, 2015, IEEE, Qingdao, China, ISBN:978-1-4799-7017-9, pp: 5670-5675. 